\title{
Effect of lateral walls on peristaltic flow through an asymmetric rectangular duct
}

\author{
Kh.S. Mekheimer ${ }^{\mathrm{a}, *}$, S.Z.-A. Husseny ${ }^{\mathrm{b}}$ and A.I. Abd el Lateef ${ }^{\mathrm{c}}$ \\ ${ }^{a}$ Mathematical Department, Faculty of Science, Al-Azhar University, Nasr City, Cairo, Egypt \\ ${ }^{\mathrm{b}}$ Mathematical Department, Faculty of Science, King AbdulAziz University, Jeddah, Saudi Arabia \\ ${ }^{\mathrm{c}}$ Basic Science Department, Higher Institute of Engineering, Shorouk City, Cairo, Egypt
}

\begin{abstract}
Peristaltic transport of an incompressible viscous fluid due to an asymmetric waves propagating on the horizontal sidewalls of a rectangular duct is studied under long-wavelength and low-Reynolds number assumptions. The peristaltic wave train on the walls have different amplitudes and phase. The flow is investigated in a wave frame of reference moving with velocity of the wave. The effect of aspect ratio, phase difference, varying channel width and wave amplitudes on the pumping characteristics and trapping phenomena are discussed in detail. The results are compared to with those corresponding to Poiseuille flow.
\end{abstract}

Keywords: Lateral walls, rectangular duct, peristaltic pumping

\section{Introduction}

It is well known that peristalsis is an important mechanism for mixing and transporting fluids, which is induced by a progressive wave of contraction or expansion moving on the wall of a channel/tube. Such mechanism occurs in the urine transport from kidney to the bladder, food mixing and chyme movement in the intestine, lymph transport in the lymphatic vessel and in vasomotion of small blood vessels, transport of spermatozoa in cervical canal, movement of eggs in the female fallopian tube and many others. Several investigators are engaged to discuss the peristaltic mechanism theoretically under various approximations [1-20]. Recently, physiologists observed that the

${ }^{*}$ Corresponding author: Kh.S. Mekheimer, Mathematical Department, Faculty of Science, Al-Azhar University, Nasr City 11884, Cairo, Egypt. E-mail: kh_mekheimer@yahoo.com. intra-uterine fluid flow due to myometrical contractions is peristaltic type motion and the myometrical contractions may occur in both symmetric and asymmetric directions, De Vries et al. [21]. In view of this, Eytan and Elad [22] have developed a mathematical model of wall-induced peristaltic fluid flow in a twodimensional channel with wave trains having a phase difference moving independently on the upper and lower walls to simulate intrauterine fluid motion in a sagittal cross-section of the uterus. They have obtained a time dependent flow solution in a fixed frame by using lubrication approach. These results have been used to evaluate fluid flow pattern in a non-pregnant uterus. They have also calculated the possible particle trajectories to understand the transport of embryo before it gets implanted at the uterine wall. Moreover, Mishra and Rao [23] investigated fluid mechanics effects of peristaltic transport in a two-dimensional asymmetric channel under the assumptions of long 
wavelength and low Reynolds number in a wave frame of reference. The channel asymmetry is produced by choosing the peristaltic wave train on the walls to have different amplitude and phase due to the variation of channel width, wave amplitudes and phase differences. More recently Kothandapani and Srinivas [24] investigated the peristaltic pumping of incompressible Newtonian fluid in an inclined asymmetric channel through a porous medium. Also, Ali and Hayat [25] investigated the peristaltic motion of an incompressible micropolar fluid in an asymmetric channel. In fact, the sagittal cross section of the uterus may be better approximated by a tube of rectangular cross section than a two-dimensional channel. The effects of the side walls on the flows in ducts with suction and injection are examined by Erdogan [26]. Tsangaris and Vlachakis [27] obtained an exact solution of the Navier-Stokes equations for a pulsating flow in a rectangular duct in order to explain the blood flow in fiber membranes used for the artificial kidney. Recently, Reddy et al. [28] studied the flow of a viscous fluid due to symmetric peristaltic waves propagating on the horizontal side walls of a rectangular duct under the assumptions of long wavelength and low Reynolds number and only studied the pumping characteristic. Literature survey indicates that such attempt is the only one for a symmetric peristaltic waves. Therefore, the present analysis is fundamental and is a second attempt in this direction. Keeping in view of the studies mentioned above, the aim of this paper is to investigate the fluid mechanics effects of peristaltic transport in a rectangular duct with asymmetric waves propagating on the horizontal side walls (lateral walls) under the assumptions of long wavelength and low Reynolds number in a wave frame of reference. The asymmetry waves are produced by choosing the peristaltic wave train on the walls to have different amplitude and phase due to the variation of channel width, wave amplitudes and phase differences.

All previous scientific investigations of transport within the biological systems they assume. The peristaltic flow in an asymmetric two-dimensional channel Explain the motion of intrauterine fluid in a sagittal cross section of the uterus. In fact, the sagittal cross section of the uterus may be better approximated by a tube of rectangular cross section than a two-dimensional channel. And the use of this method of treatment will help to improve the overall performance of the artificial heart and blood vessel.

\section{Formulation of the problem}

Consider the motion of an incompressible viscous fluid in a duct of rectangular cross section with width $2 d_{3}$ and height $d_{1}+d_{2}$ as shown in Fig. 1. Cartesian coordinate system $(\mathrm{X}, \mathrm{Y}, \mathrm{Z})$ is with $\mathrm{X}, \mathrm{Y}$ and $\mathrm{Z}$ axes corresponding to axial, lateral, and vertical directions, respectively, of a rectangular duct. The duct walls are flexible, and an infinite train of sinusoidal waves propagate with constant velocity $\mathrm{c}$ only along the walls parallel to the XY plane in the axial direction. The peristaltic waves on the walls are given by

$$
\begin{array}{ll}
Z=H_{1}=d_{1}+a_{1} \cos \frac{2 \pi}{\lambda}(X-c t) & \text { upper wall } \\
\left.Z=H_{1}=-d_{2}-b_{1} \cos \left(\frac{2 \pi}{\lambda}(X-c t)\right)+\phi\right) & \text { lower wall }
\end{array}
$$

where $a_{1}, b_{1}$ are the amplitudes of the waves, $\lambda$ is the wave length, $d_{1}+d_{2}$ is the width of the channel, the phase difference, $\phi$ varies in the range $0 \leqslant \phi \leqslant \pi$, $\phi=0$ corresponds to symmetric channel with waves out of phase and $\phi=\pi$ the waves are in phase, and further $a_{1}, b_{1}, d_{1}, d_{2}$ and $\phi$ satisfies the condition $a_{1}^{2}+$ $b_{1}^{2}+2 a_{1} b_{1} \cos \left(d_{1}+d_{2}\right)^{2}$. Introducing a wave frame $(x, y, z)$ moving with velocity $\mathrm{c}$ away from the fixed frame $(X, Y, Z)$ by the transformation between the two frames is given by

$x=X-c t, y=Y, z=Z, u=U-c$,

$w=W, v=V, p(x, z)=P(X, Z, t)$

where $(u, v, w)$ and $(U, V, W)$ are the velocity components, and $\mathrm{p}$ and $\mathrm{P}$ are the pressures in wave and fixed frames of reference, respectively. Using the nondimensional quantities

$$
\begin{aligned}
& \bar{x}=\frac{x}{\lambda}, \bar{y}=\frac{y}{d_{3}}, \bar{z}=\frac{z}{d_{1}}, \bar{u}=\frac{u}{c}, \bar{w}=\frac{w}{c \delta}, \bar{v}=\frac{d_{3}}{c \lambda \delta^{3}} v \\
& \bar{t}=\frac{c t}{\lambda}, h_{1}=\frac{H_{1}}{d_{1}}, h_{2}=\frac{H_{2}}{d_{1}}, d=\frac{d_{2}}{d_{1}}, a=\frac{a_{1}}{d_{1}}, b=\frac{b_{1}}{d_{1}}, \\
& R_{e}=\frac{c d_{1}}{v}, \beta=\frac{d_{1}}{d_{3}}, \bar{p}=\frac{d_{1}^{2} p}{\mu c \lambda} .
\end{aligned}
$$

in the Navier-Stokes equations and equation of continuity, which governs the flow, we get (dropping the bars) 


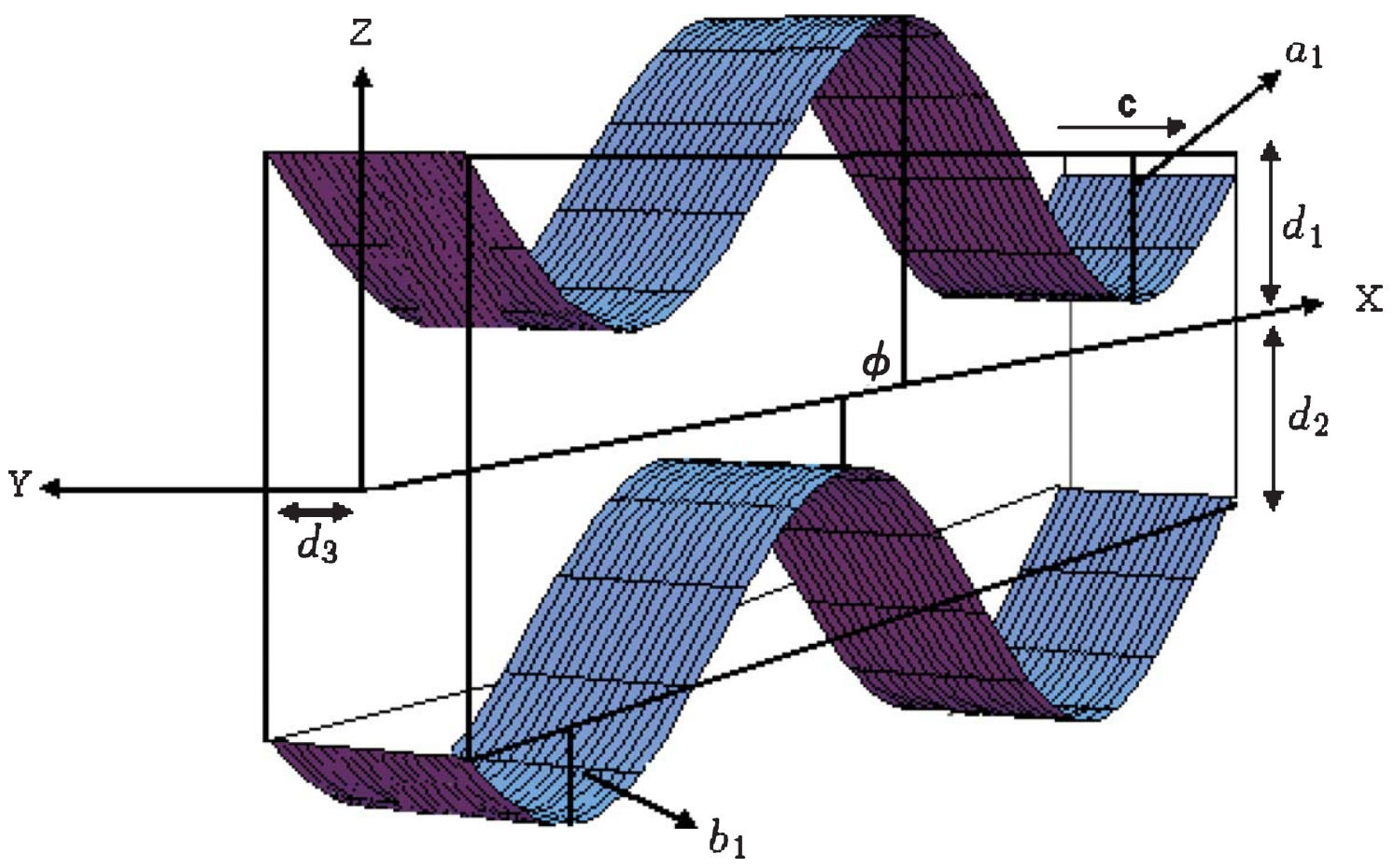

Fig. 1. Geometry of the problem

$$
\begin{aligned}
& R_{e} \delta\left[u \frac{\partial u}{\partial x}+\delta \beta^{2} v \frac{\partial u}{\partial y}+w \frac{\partial u}{\partial z}\right]=-\frac{\partial p}{\partial x}+\delta^{2} \frac{\partial^{2} u}{\partial x^{2}}+\beta^{2} \frac{\partial^{2} u}{\partial y^{2}}+\frac{\partial^{2} u}{\partial z^{2}} \\
& R_{e} \delta^{4}\left[u \frac{\partial v}{\partial x}+\delta \beta^{2} v \frac{\partial v}{\partial y}+w \frac{\partial v}{\partial z}\right]=-\frac{\partial p}{\partial y}+\delta^{3}\left(\delta^{2} \frac{\partial^{2} v}{\partial x^{2}}+\beta^{2} \frac{\partial^{2} v}{\partial y^{2}}+\frac{\partial^{2} v}{\partial z^{2}}\right) \\
& R_{e} \delta^{3}\left[u \frac{\partial w}{\partial x}+\delta \beta^{2} v \frac{\partial w}{\partial y}+w \frac{\partial w}{\partial z}\right]=-\frac{\partial p}{\partial z}+\delta^{4} \frac{\partial^{2} w}{\partial x^{2}}+\delta^{2}\left(\beta^{2} \frac{\partial^{2} w}{\partial y^{2}}+\frac{\partial^{2} w}{\partial z^{2}}\right)
\end{aligned}
$$$$
\frac{\partial u}{\partial x}+\delta \beta^{2} \frac{\partial v}{\partial y}+\frac{\partial w}{\partial z}=0
$$

where $\beta$ is an aspect ratio and $R_{e}$ is a Reynolds number, the aspect ratio $\beta<1$ means that height is less compared to width, and $\beta=0$ corresponds to a two dimensional channel. When $\beta=1$, the rectangular duct becomes a square duct and for $\beta>1$, the height is more compared to width. Under lubrication approach (negligible inertia $R_{e} \rightarrow 0$ and long wavelength $\delta \ll 1$ ) the Eqs. (4)-(7) reduce to

$$
\begin{aligned}
& \beta^{2} \frac{\partial^{2} u}{\partial y^{2}}+\frac{\partial^{2} u}{\partial z^{2}}=\frac{d p}{d x} \\
& \frac{\partial u}{\partial x}+\frac{\partial w}{\partial z}=0
\end{aligned}
$$

The corresponding no-slip boundary conditions are

$$
u=-1 \text { at } y= \pm 1
$$




$$
u=-1 \text { at } z=h_{1}, z=h_{2},
$$

where $h_{1}=1+a \cos (2 \pi x), h_{2}=-d-b \cos (2 \pi x+\phi)$, $0 \leq a \leq 1, a=0$ for straight duct and $a=1$ corresponds to total occlusion. Introducing $x=x, y=\beta \dot{y}$, $z=z$ in Eqn .(8), we get

$$
\frac{\partial^{2} u}{\partial \hat{y}^{2}}+\frac{\partial^{2} u}{\partial \dot{z}^{2}}=\frac{d p}{d \dot{x}}
$$

Substituting $u=u ́-1+1 \frac{1}{2} \frac{d p}{d x}\left(h_{1}-z\right)\left(h_{2}-z\right)$, in (12), we get

$$
\frac{\partial^{2} \dot{u}}{\partial \dot{y}^{2}}+\frac{\partial^{2} \dot{u}}{\partial \bar{z}^{2}}=0
$$

The corresponding boundary conditions are

$$
\begin{array}{ll}
\dot{u}=-\frac{1}{2} \frac{d p}{d x}\left(h_{1}-\dot{z}\right)\left(h_{2}-\dot{z}\right) & \text { at } \dot{y}= \pm \frac{1}{\beta} \\
\dot{u}=0 & \text { at } z=h_{1}, \dot{z}=h_{2}
\end{array}
$$

The solution of (8) using Eqs. (12)-(15), valid in $-1 \leq y \leq 1, h_{2} \leq z \leq h_{1}$, satisfying the corresponding boundary conditions (10) and (11) is given by

$$
\begin{aligned}
u= & -1+\frac{d p}{d x}\left(\frac{\left(h_{1}-z\right)\left(h_{2}-z\right)}{2}-\frac{2}{\left(h_{1}-h_{2}\right)}\right. \\
& \left.\times \sum_{n=1}^{\infty} \frac{\left((-1)^{n}-1\right) \cosh \left(\frac{\alpha_{n}}{\beta} y\right) \sin \alpha_{n}\left(z-h_{2}\right)}{\alpha_{n}^{3} \cosh \left(\frac{\alpha_{n}}{\beta}\right)}\right)
\end{aligned}
$$

where $\alpha_{n}=(n \pi) /\left(h_{1}-h_{2}\right)$ The volumetric flow rate in the rectangular duct in the wave Frame (in vertical half) is given by
The instantaneous flux in the laboratory frame is

$Q=\int_{h_{2}}^{h_{1}} \int_{0}^{1}(u+1) d y d z=q+\left(h_{1}-h_{2}\right)$

The average flux $\bar{Q}(x, t)$ over one period $(T=\alpha / c)$ of the peristaltic wave is

$\bar{Q}=\frac{1}{T} \int_{h_{2}}^{h_{1}} \int_{0}^{1}(Q) d y d z=q+1+d$

From Eqs (17) and (19), the pressure gradient is obtained as

$\frac{d p}{d x}=\frac{(\bar{Q}-1-d)+\left(h_{1}-h_{2}\right)}{-\frac{\left(h_{1}-h_{2}\right)^{3}}{12}+\sum_{n=1}^{\infty} \frac{4 \beta\left(1-(-1)^{n}\right) \tanh \frac{\alpha_{n}}{\beta}}{\left(h_{1}-h_{2}\right) \alpha_{n}^{5}}}$

Integrating Eq. (20) with respect to $x$ over one wavelength, we get the pressure rise (drop) over one cycle of the wave as

$$
\Delta p=(\bar{Q}-1-d) I_{1}+I_{2}
$$

where

$$
\begin{aligned}
& I_{1}=\int_{0}^{1} \frac{1}{\frac{-\left(h_{1}-h_{2}\right)^{3}}{12}+\frac{4 \beta}{\left(h_{1}-h_{2}\right)} \sum_{n=1}^{\infty} \frac{\left(1-(-1)^{n}\right) \tanh \frac{\alpha_{n}}{\beta}}{\alpha_{n}^{5}}} d x \\
& I_{2}=\int_{0}^{1} \frac{\left(h_{1}-h_{2}\right)}{\frac{-\left(h_{1}-h_{2}\right)^{3}}{12}+\frac{4 \beta}{\left(h_{1}-h_{2}\right)} \sum_{n=1}^{\infty} \frac{\left(1-(-1)^{n}\right) \tanh \frac{\alpha_{n}}{\beta}}{\alpha_{n}^{5}}} d x
\end{aligned}
$$

The stream function associated with the flow depends on all the three coordinates. We can find the streamlines in $x z$ plane (fixing a lateral station $y$ )and with the condition. $\psi=0$ at $z=1 / 2\left(h_{1}+h_{2}\right)$ will take the form

$$
\begin{aligned}
\psi(x, y)= & \frac{1}{2}\left(h_{1}+h_{2}\right)-z+\frac{d p}{d x}\left\{\frac{1}{24}\left(h_{1}+h_{2}-2 z\right)\left(h_{1}^{2}-4 h_{1} h_{2}+h_{2}^{2}+2\left(h_{1}+h_{2}\right) z-2 z^{2}\right)\right. \\
& \left.+\sum_{n=1}^{\infty} \frac{\left(1-(-1)^{n}\right) \cosh \left(\frac{\alpha_{n}}{\beta} y\right)}{\alpha_{n}^{4} \cosh \left(\frac{\alpha_{n}}{\beta}\right)}\left(\sqrt{\frac{1}{2}\left((-1)^{n}+1\right)} \cos \left(\alpha_{n}\left(z-h_{2}\right)\right)\right)\right\}
\end{aligned}
$$

In the limit $\beta \rightarrow 0$ (keeping a fixed and $d \rightarrow \infty$ ), the rectangular duct reduces to a two-dimensional channel and our results reduce to those given by Mishra and Rao [23], also when $\left(d_{1}=d_{2}, a_{1}=b_{1}\right)$ and $\phi=0$ (symmetric walls) our results reduce to those given by Reddy, et al. [28]. Finally, for the symmetric walls and $\beta \rightarrow 0$ our results are the same as those given by Shapiro et al. [1]. 
(i)

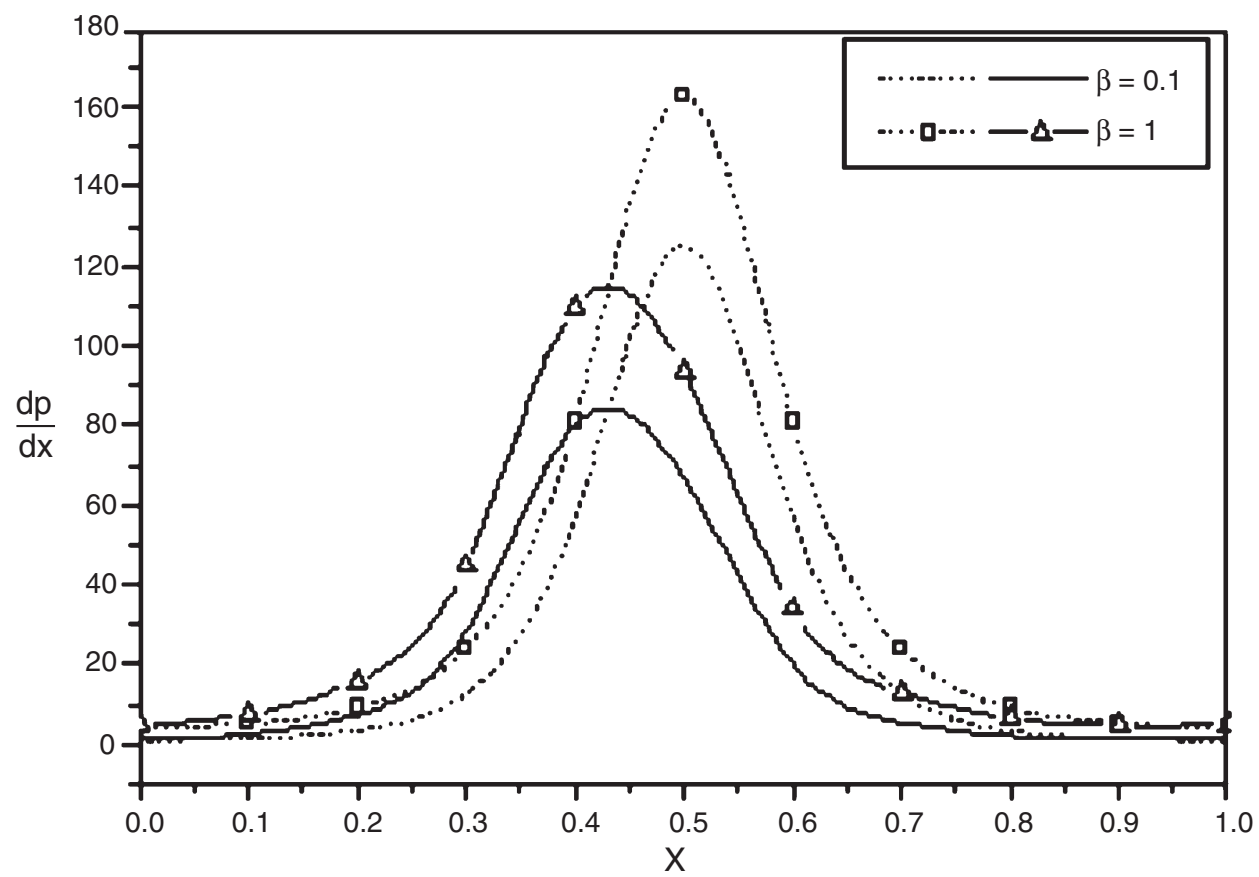

(ii)

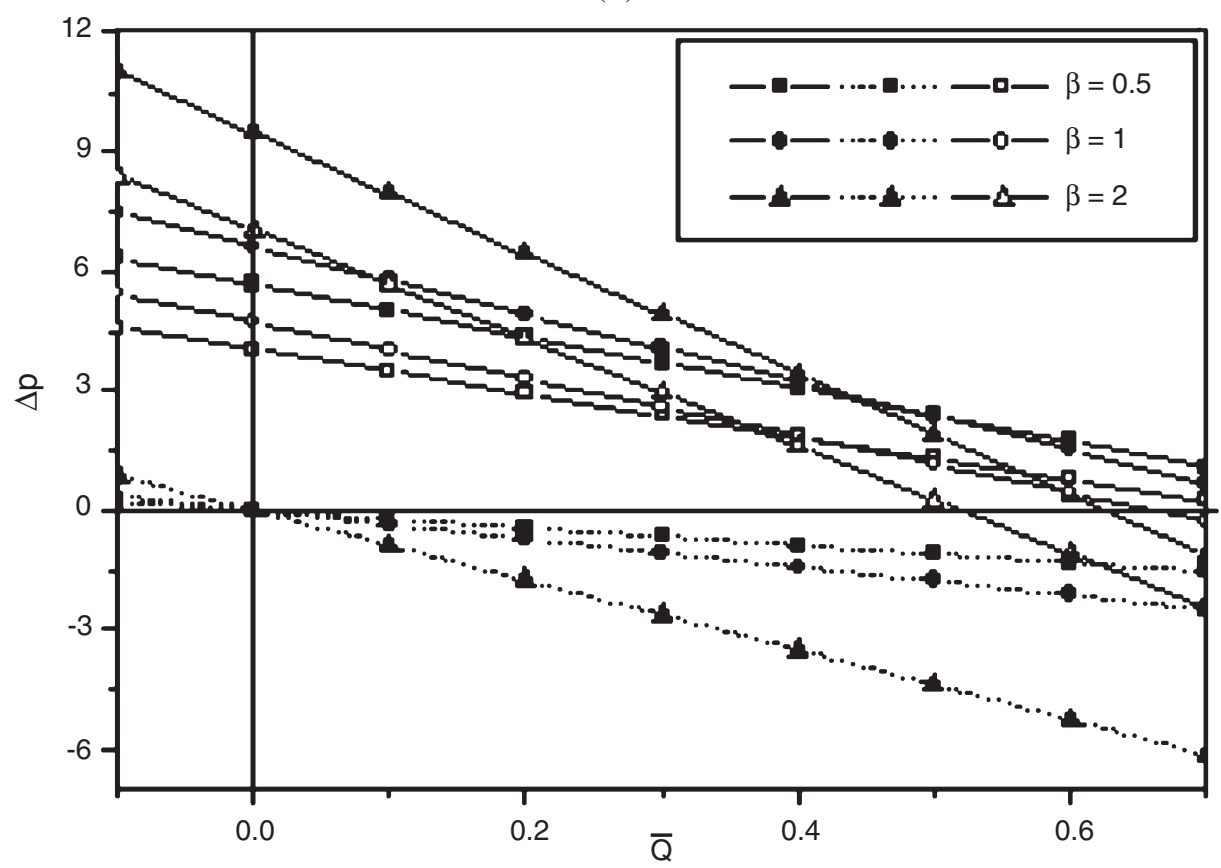

Fig. 2. (i) Variation of $d p / d x$ versus $x$ for different values of $\beta$ with $a=b=0.6$ and (i) $\phi=0$ (dot lines), (ii) $\phi=\pi / 4$ (solid lines). (ii) Variation of $\Delta p$ versus $\bar{Q}$ for different values of $\beta$ with $a=b=0.6$ and (i) $\phi=0$ (solid lines with closed mark), (ii) $\phi=\pi / 4$ (solid lines with open mark), (iii) $\phi=\pi$ (dot lines). 
(i)

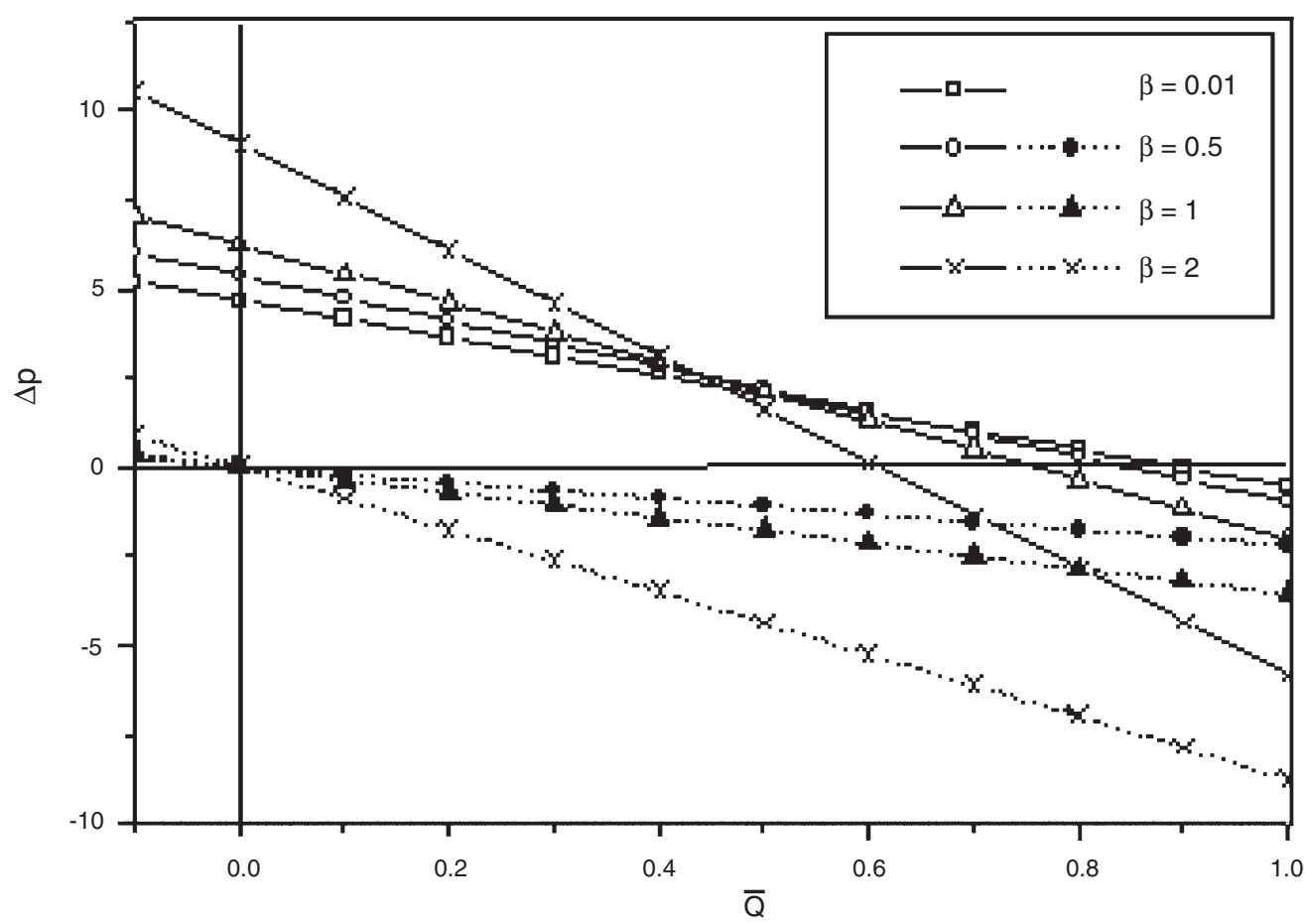

(ii)

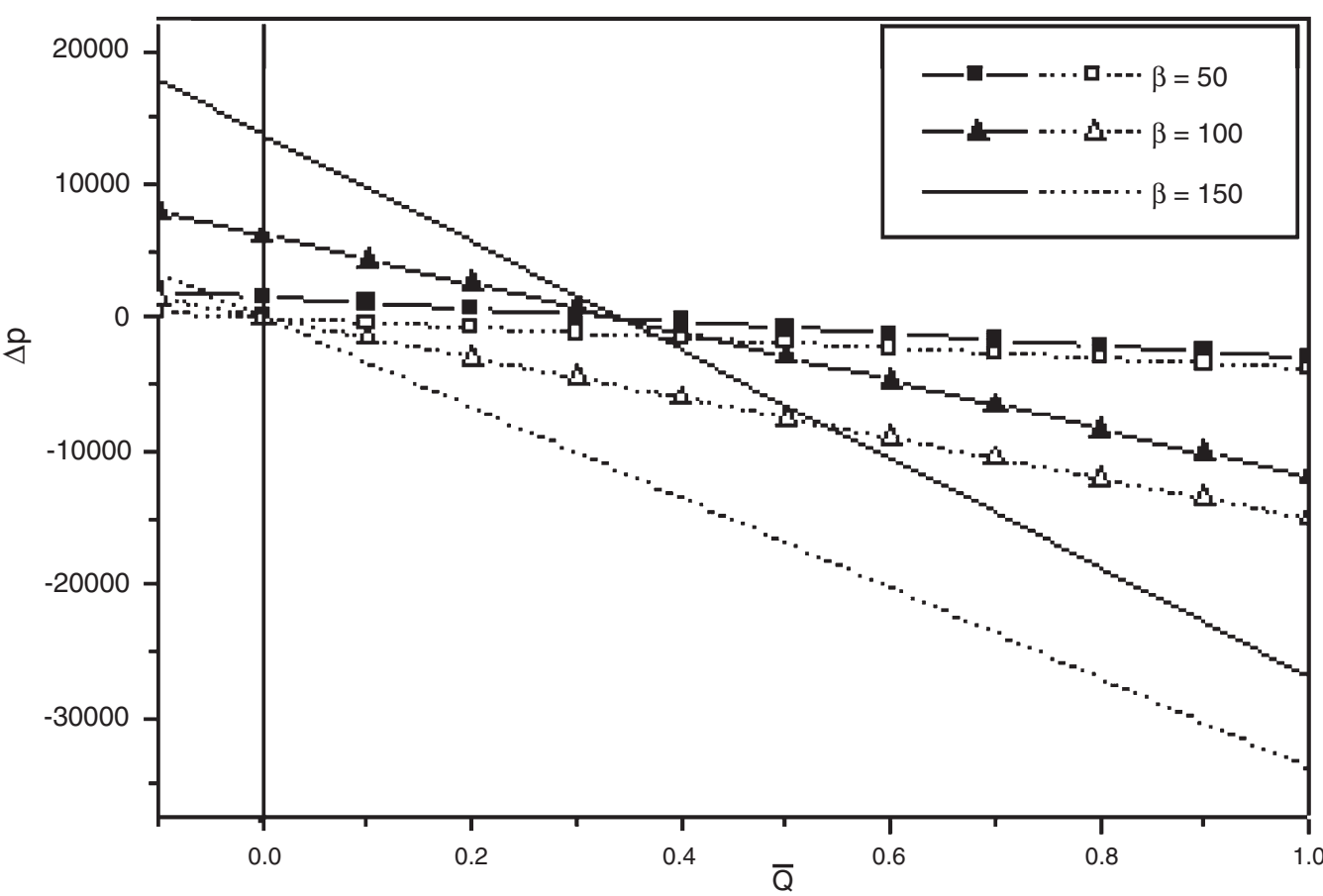

Fig. 3. Variation of $\Delta p$ verus $\bar{Q}$ with $a=b=0.6$ and $\phi=\pi / 4$ (i) for small values of (ii) For large $\beta$, and the corresponding graphs for Poiseuille $(a=b=0)$ flow are given in dot lines. 
(i)

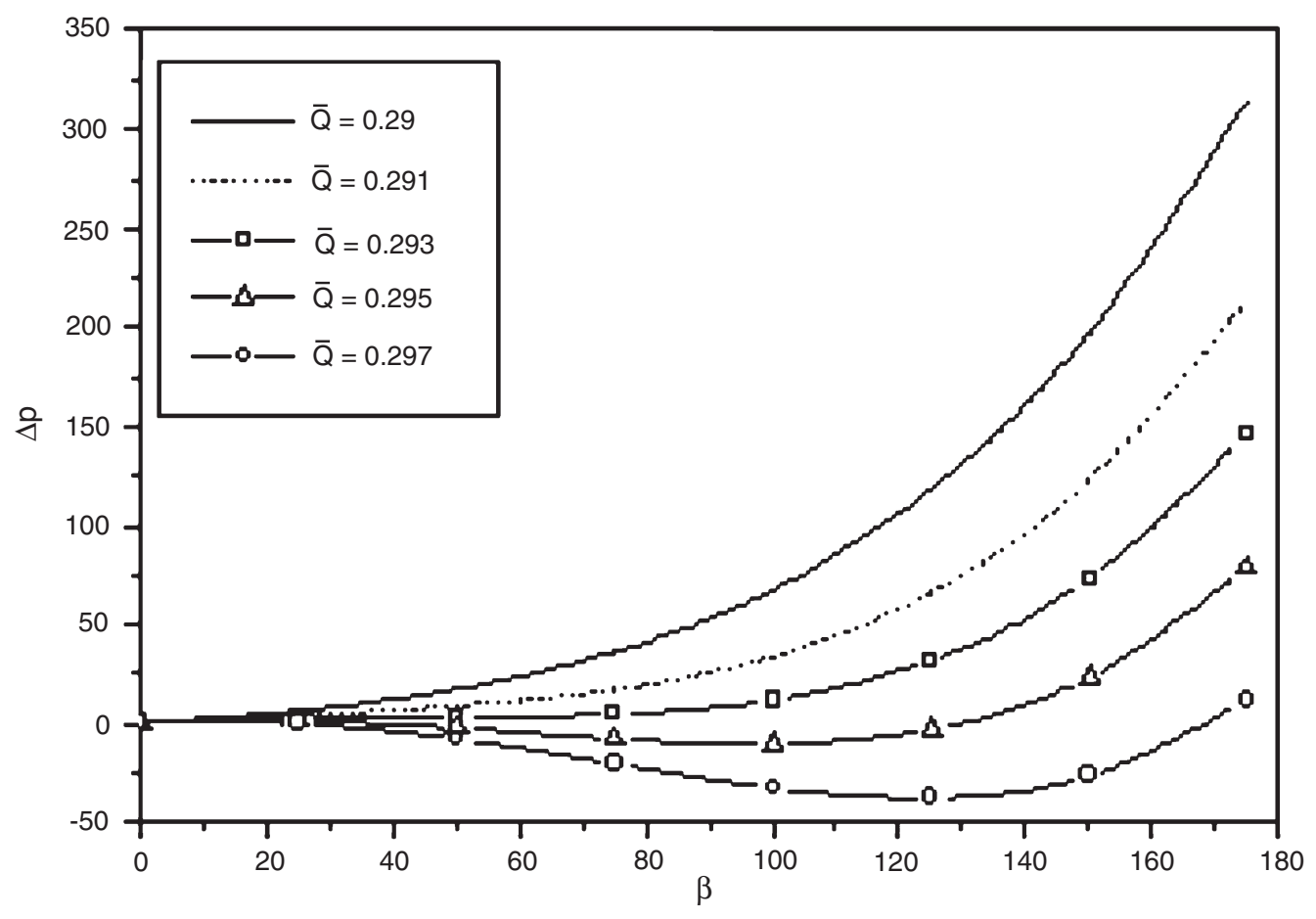

(ii)

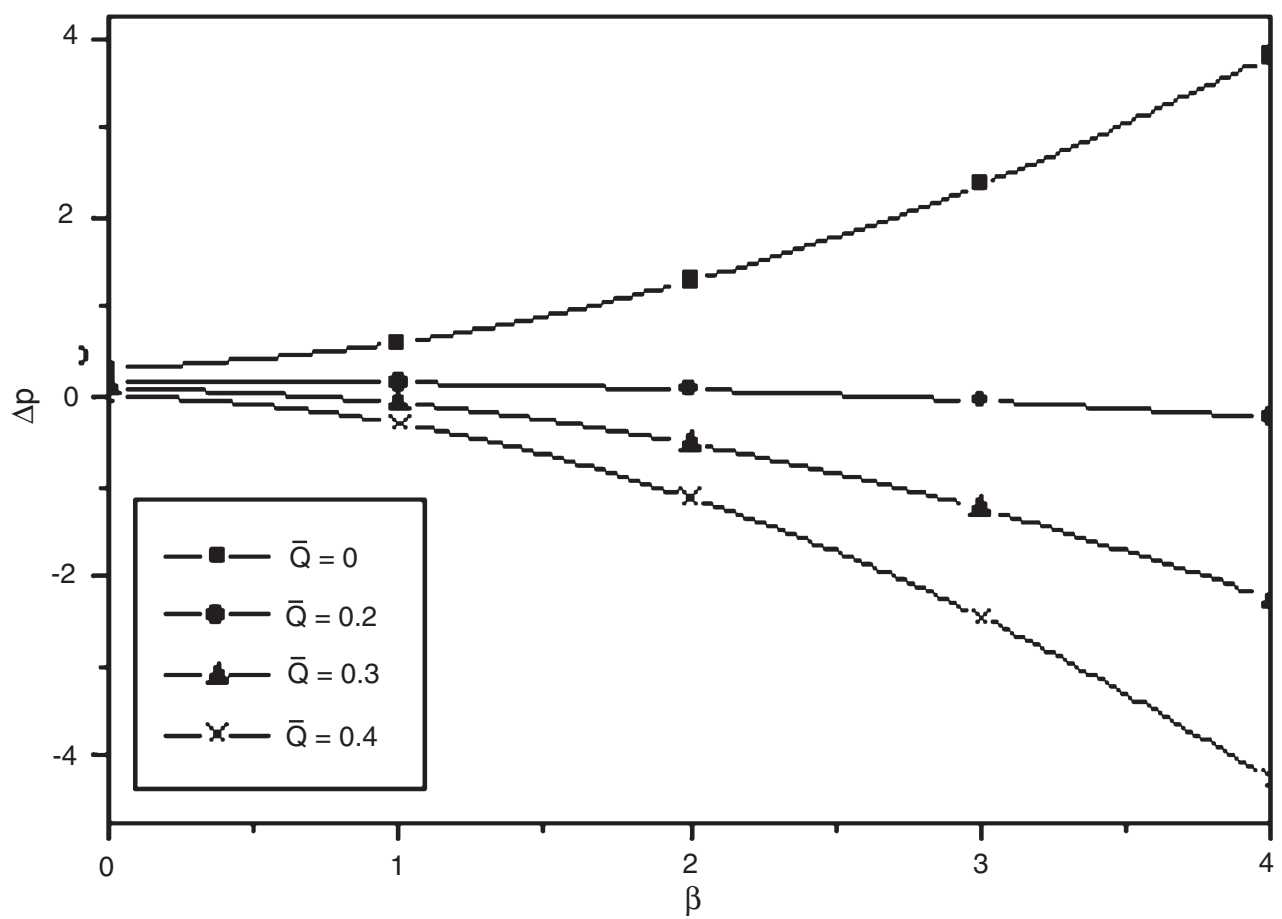

Fig. 4. Variation of $\Delta p$ versus $\beta$ with $a=b=0.7$ and $\phi=\pi / 4$ (i) for different values of flux $\bar{Q}$ and (ii) enlargement of (i) near $\beta=0$. 


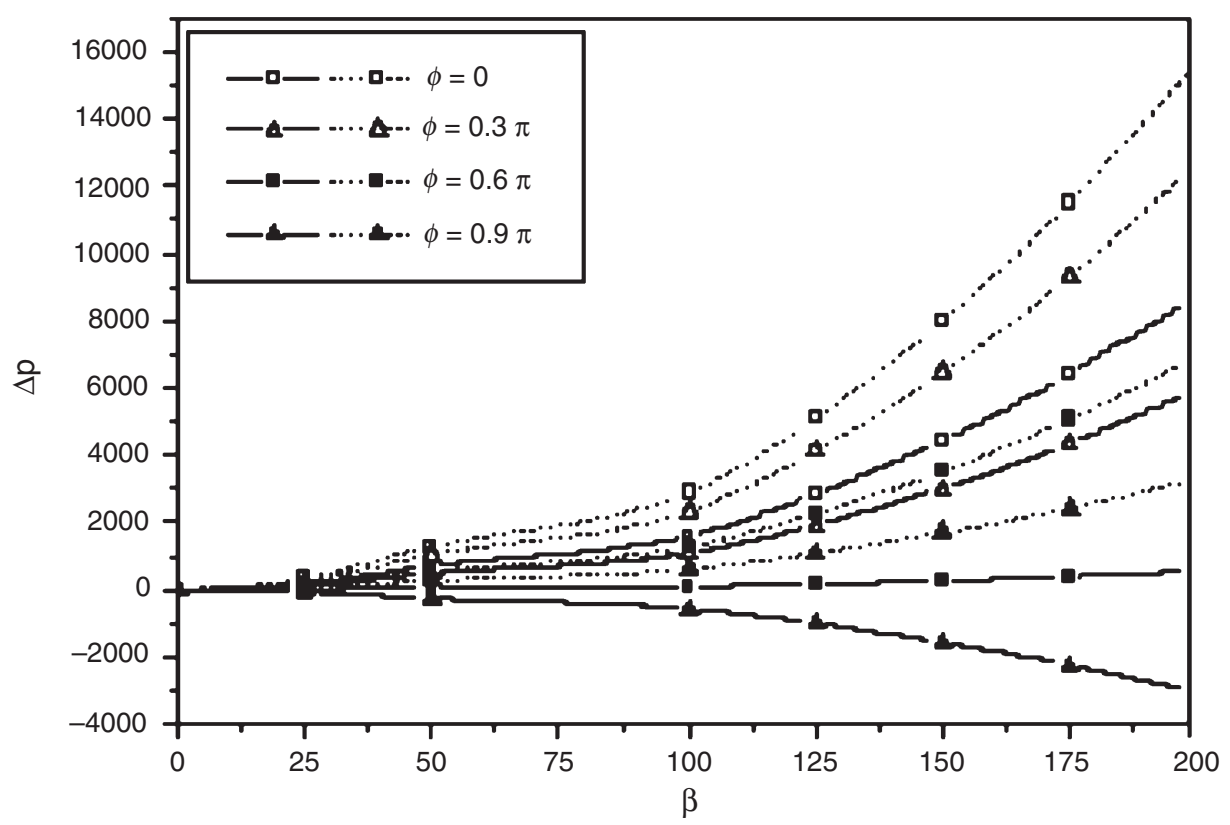

Fig. 5. variation of $\Delta p$ versus $\beta$ with $a=b=0.7, d=2$ and $\bar{Q}=0.1$ (solid lines) and ( $\bar{Q}=-0.1$ dot lines) for different phase.

\section{Discussion}

This section describes the influence of various emerging parameters of the problem on $d p / d x, \Delta p, \bar{Q}$ and trapping phenomena. Moreover, to understand the effect of lateral asymmetric walls on the pumping characteristic one have to analyze the pressure-flux relation given in Eq. (21) for different values of the aspect ratio $\beta$ and the dimensional phase shift $\phi$. Due to the complexity of expression (21), the integrations and the series involved are not integrable in closed form, so numerical integrations are performed and the graphical results are presented. Physically, as $\beta$ increases from small value to a value greater than one, a transition takes place from rectangular duct with domination of width to the one with height passing through a crosssection of square duct. In Fig. 2(i) the variation of $d p / d x$ within a wavelength for different values of the phase different $\phi$ and the aspect ratio $\beta$ is presented. It is shown that as $\phi$ increases, i.e moves from symmetric to an asymmetric wall waves, the pressure gradient decreases, while as $\beta$ increases the pressure gradient increases. Figure 2(ii) and (3) depicts the pumping curves with a fixed value of $\mathrm{a}=\mathrm{b}=0.6$ and for different values of $\beta$ and $\phi$. For small values of $\beta$, any two pumping curves intersect at a point in the first quadrant as seen in Fig. 3(i). As long as this point remain in the first quadrant, to the left of this point pumping increases and to the right both pumping and free pumping $\Delta p=0$ decreases with increasing $\beta$, i.e. vertical dimension a is large compared to horizontal dimension $d$. This point of intersection will stay at the first quadrant as $\phi$ increases but with less pressure rise, while this point move to the fourth quadrant for large values of $\beta$ as shown in Fig. 3(ii) and so, both pumping and free pumping increases with increasing $\beta$ while it decreases as $\phi$ increases. Our results for the pumping characteristics $(\Delta p$ versus $\bar{Q})$ are comparing to those of Poiseuille (without peristaltic $a=b=0$ ) flow in dot lines in Fig. 3(i), (ii). The variation of $\Delta p$ with $\beta$ for different values of $\bar{Q}$ is illustrated in Fig. 4(i), (ii). It is observed that $\Delta p$ increases for small values of $\bar{Q}$. Fig. 4(ii) is an enlargement of Fig. 4(i), showing that the curves for various $\bar{Q}$ do not meet at a single point near $\beta=0.0$. Figure 5 depicts the variation of $\Delta p$ with $\beta$ and for different values of $\phi$. This figure shows that $\Delta p$ decreases as $\phi$ increases while it increases as $\beta$ increases for small values of $\phi$. The variation of $\bar{Q}$ with $\beta$ for different values of $\Delta p$ and $a, b$ (amplitudes of peristaltic waves on the upper and lower walls) is depicted in Fig. 6, where the flow rate $\bar{Q}$ decreases for $\Delta p=-1$ and increases for $\Delta p=4$ only for small values of $\beta$. Also, Fig.6(i), (ii) shows that the free pumping flux rate $\bar{Q}$ attain a minimum around $\beta \simeq 0.25$ and then have approximately 
(i)

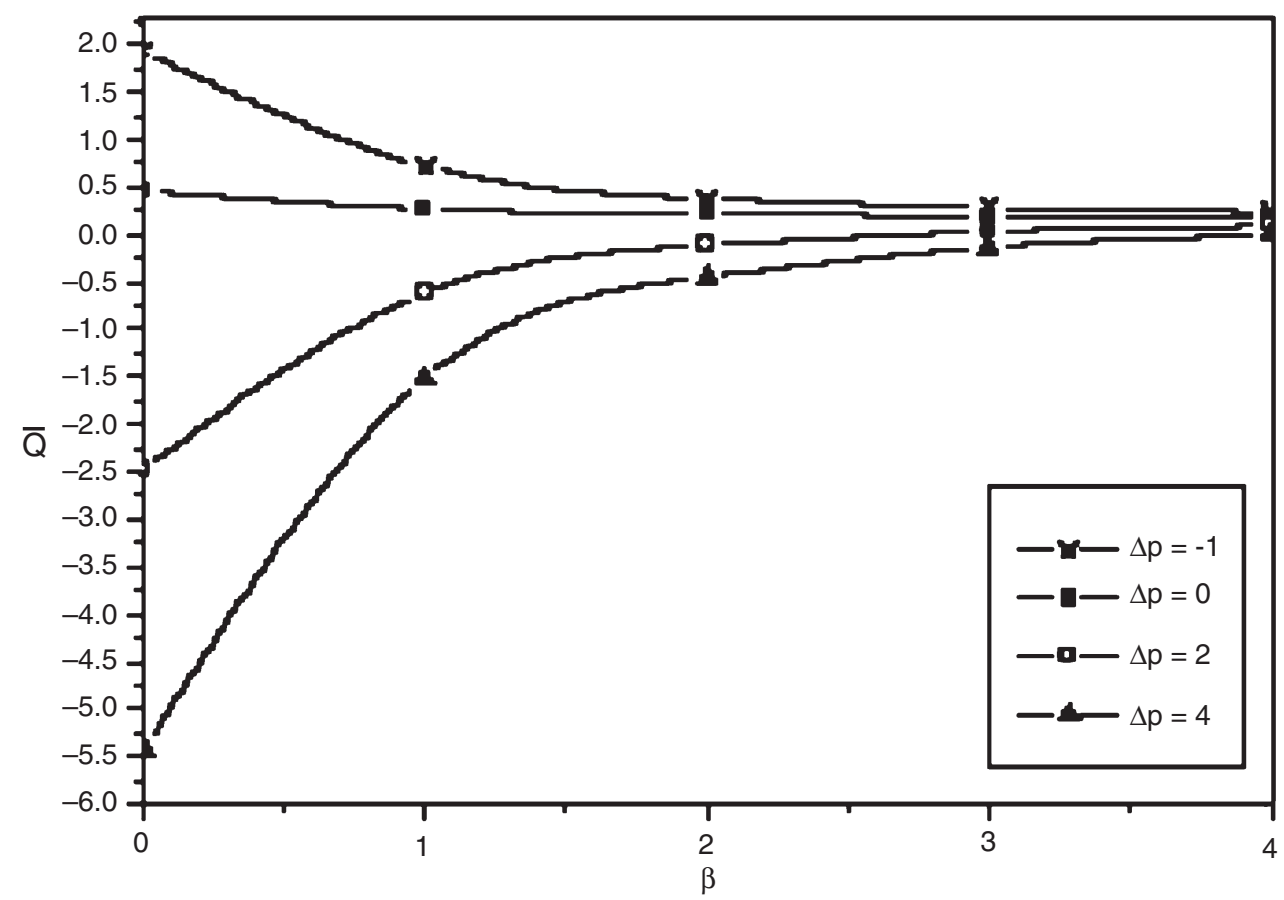

(ii)

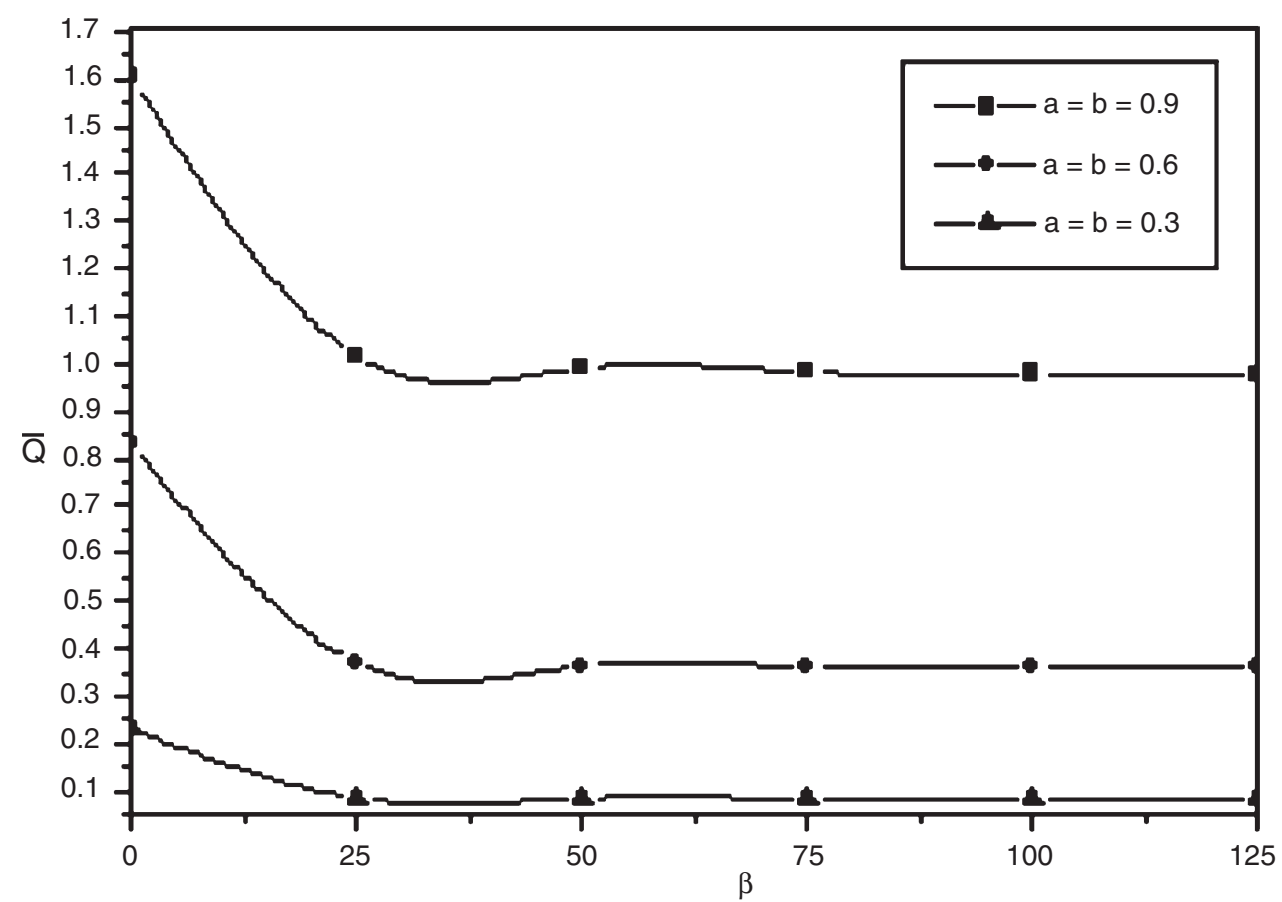

Fig. 6. (i) Variation of $\bar{Q}$ versus $\beta$ with $a=b=0.7, \mathrm{~d}=2$ and $\phi=\pi / 2$ for different values of $\Delta p$, and (ii) variation of $\bar{Q}$ versus $\beta$ with free pumping $(\Delta p=0)$ for different $a, b$ at $d=1$ and $\phi=\pi / 5$. 
(i)

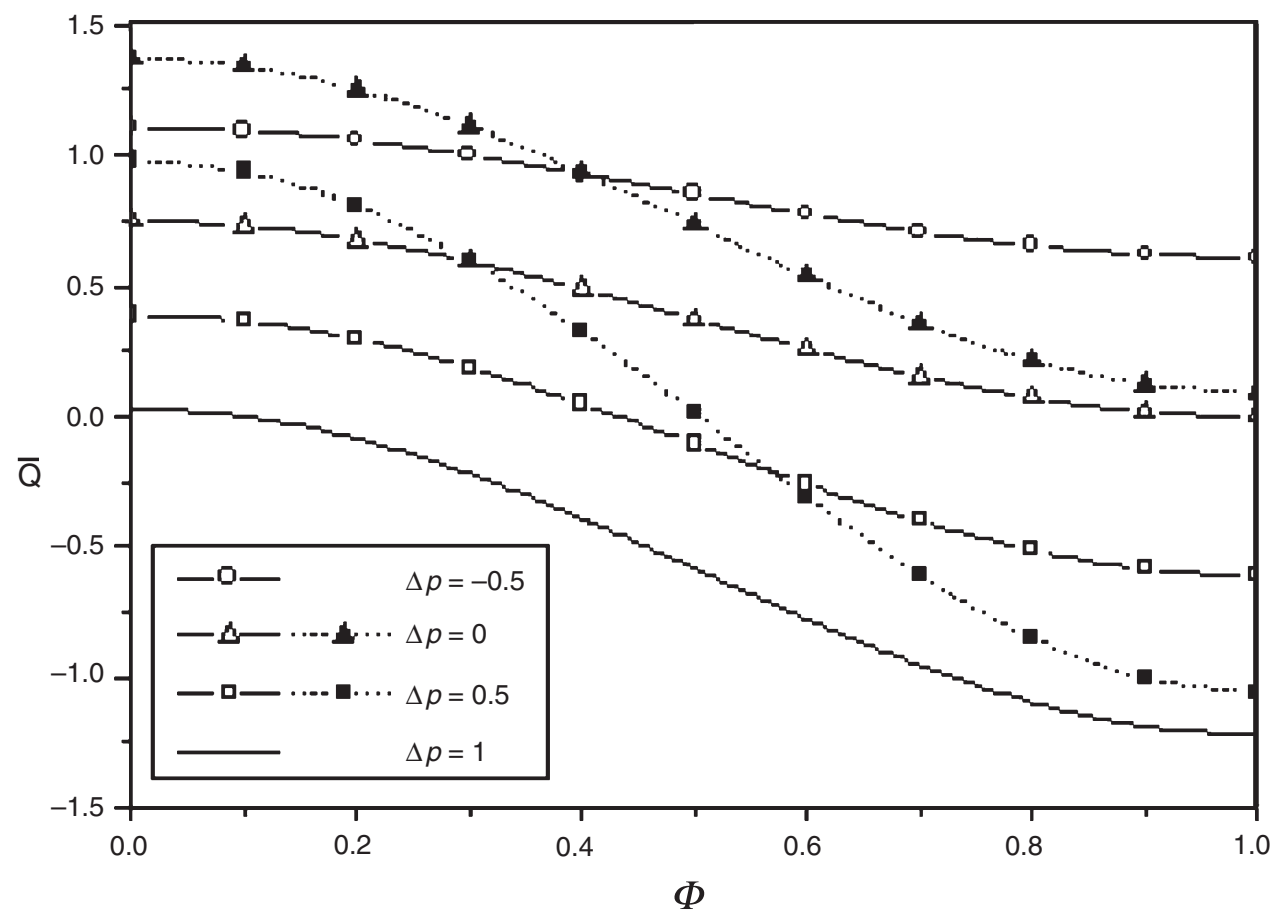

(ii)

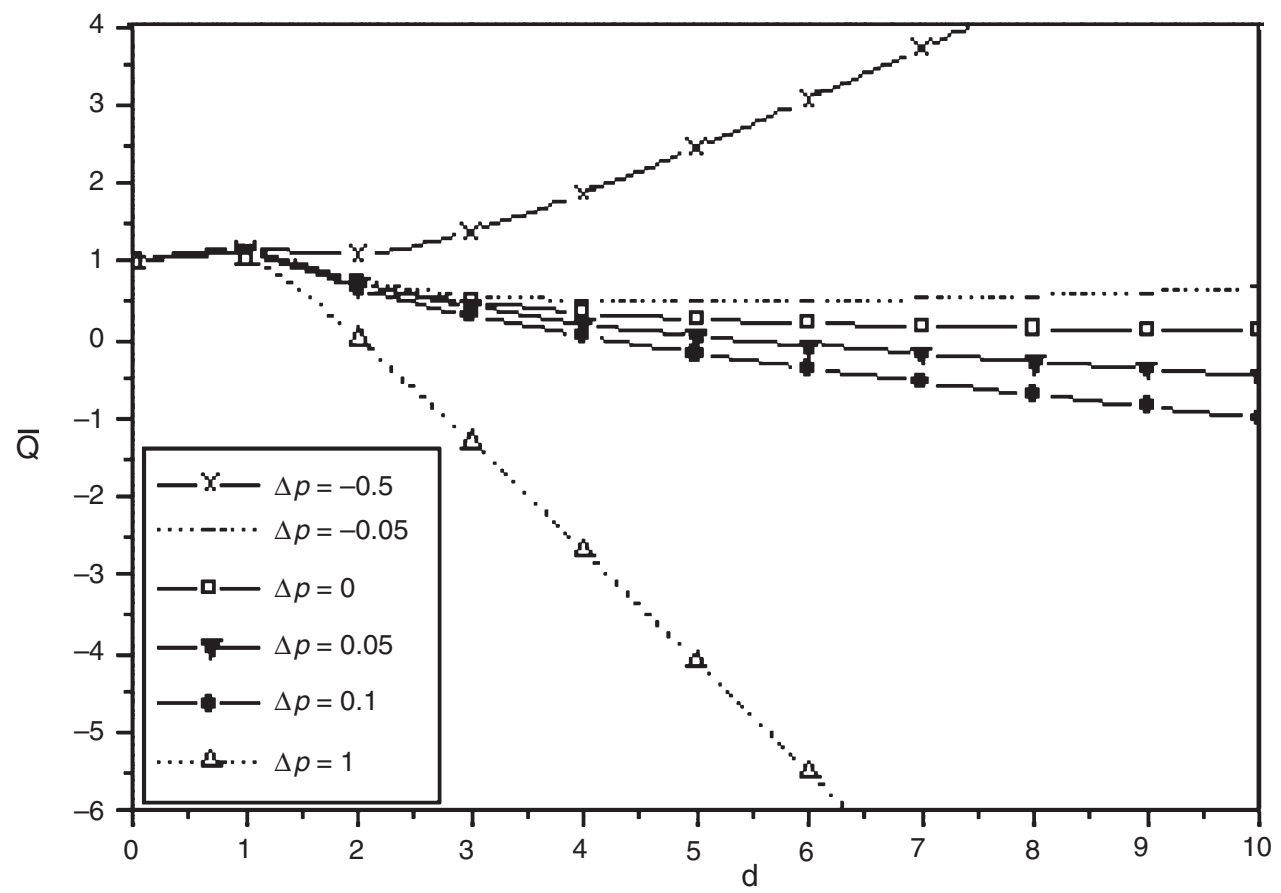

Fig. 7. (i) Variation of $\bar{Q}$ with $\phi$ when $d=2, \beta=0.5$ for different values of $\Delta p$ at $a=b=0.7$ (solid lines) and $a=0.7$; $b=1.2$ (dot lines). (ii) Variation of $\bar{Q}$ with $d$ for different $\Delta p$ with $a=0.7, b=1.2 \beta=0.5$, and $\phi=\pi / 2$. 


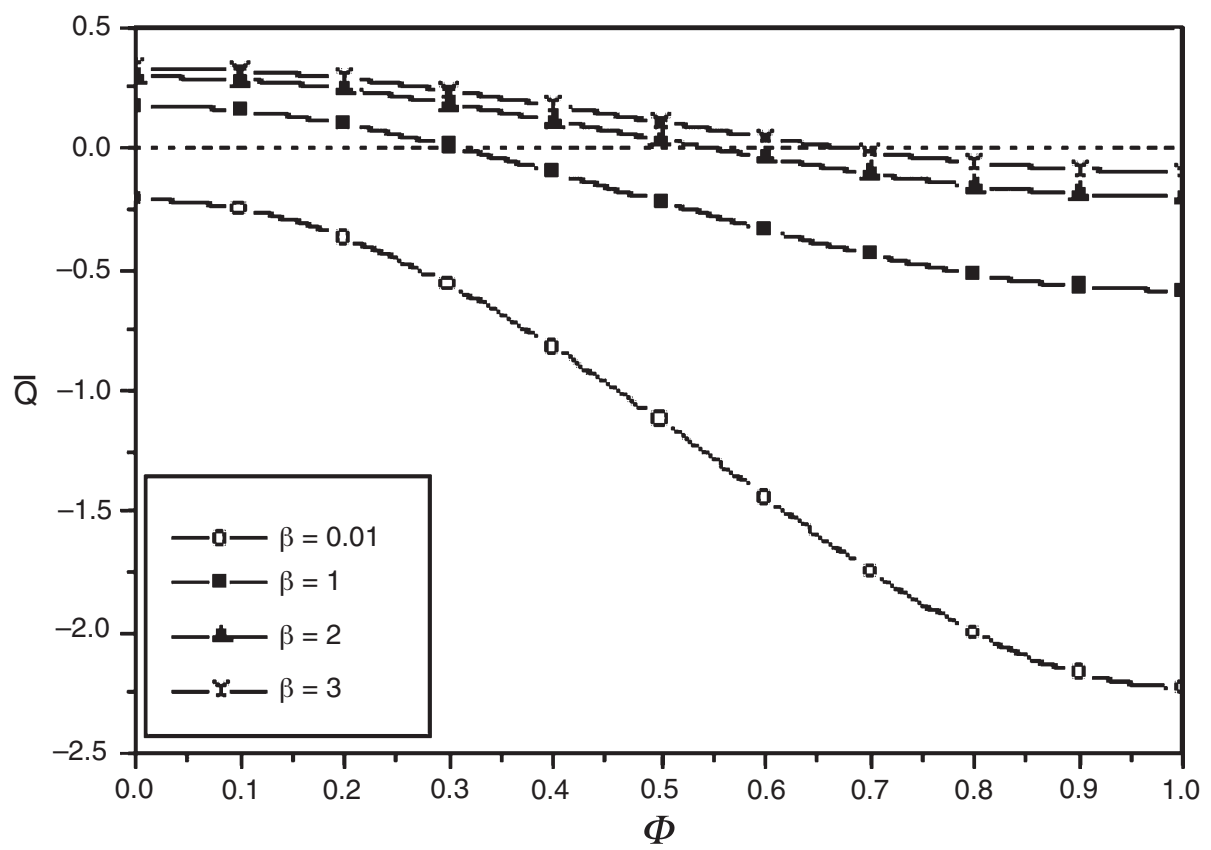

Fig. 8. Variation of $\overline{\mathrm{Q}}$ versus $\phi$ with $a=b=0.7, \mathrm{~d}=2$ and $\Delta \mathrm{p}=1$ for different $\beta$.

constant value and this flow rate decrease as the amplitudes of peristaltic waves increases. The variation of the time - average flux $\bar{Q}$ as a function $\phi$ and $d$ (width of the channel) for different values of $\Delta p$ is presented in Fig. 7(i), (ii) respectively. We observe that when $\phi=0$, the time - average flux $\bar{Q}$ is maximum and decreases as $\phi$ increases then vanishes for certain value of $\phi$ and remains negative until $\phi$ becomes 1 . When $\Delta p=0$, for free pumping case, we observe $\bar{Q}$ is zero for $\phi=1$ (i.e. when peristaltic waves are in phase, the cross section of the channel remains same through out) and is positive for all $0 \leq \phi<1.1$. Also, $\bar{Q}$ remains always positive for $\Delta p<0$ (copumping region) as pressure assists the flow due to peristalsis on the walls. Fig.7(ii) shows that for $\Delta p \geq 0$ the flux rate decreases as the distance $d$ between the walls increases due to the reduction in the peristalsis effects. From the curve $\Delta p=-0.5$ $(\Delta p<0), \bar{Q}$ decreases for some d (small) in the beginning, but it start increasing for d large as the Poiseuille flow due to the pressure loss dominates the peristaltic flow. Figure 8 show the variation of $\bar{Q}$ with $\phi$ for different values of $\beta$. It is observed that as $\phi$ increases the flow rate flux decreases (more rapidly for small values of $\beta$ ), and the change in $\bar{Q}$ is small for large values of $\beta$.

\section{Trapping}

In the wave frame the streamlines in general have a shape similar to the walls as the walls are stationary. But under certain conditions some streamlines split (due to the existence of a stagnation point) to enclose a bolus of fluid particles in closed streamlines. In the fixed frame the bolus moves as a whole at the wave speed as if trapped by the wave to see the effects of lateral station $\mathrm{y}$, aspect ratio $\beta$ and phase difference $\phi$ on the trappings, we prepared Figures 9-11. Figure 9 reveals that in the symmetric $(\phi=0)$ channel the trapping is about the center line and trapped bolus decrease in size as the lateral state $y$ increases. The effect of the aspect ratio $\beta$ on the trapping is observed in Fig. 10, where as $\beta$ increases the bolus size increases and for $\beta=1$, the bolus will move as the wave wall. The effect of phase shift $\phi$ on trapping with same amplitudes $\mathrm{a}=\mathrm{b}=0.5$ for ${ }^{-} \mathrm{Q}=1.4$ (within the centerline trapping limits) is illustrated in Fig. 11. It is observed that the bolus appearing in the center region for $\phi=0$ then moves towards left and decreases in size as $\phi$ increases. For $\phi=\pi$ the bolus disappears and the streamlines are parallel to the boundary walls $[21,23]$ and here $\bar{Q}>0$ is pos- 


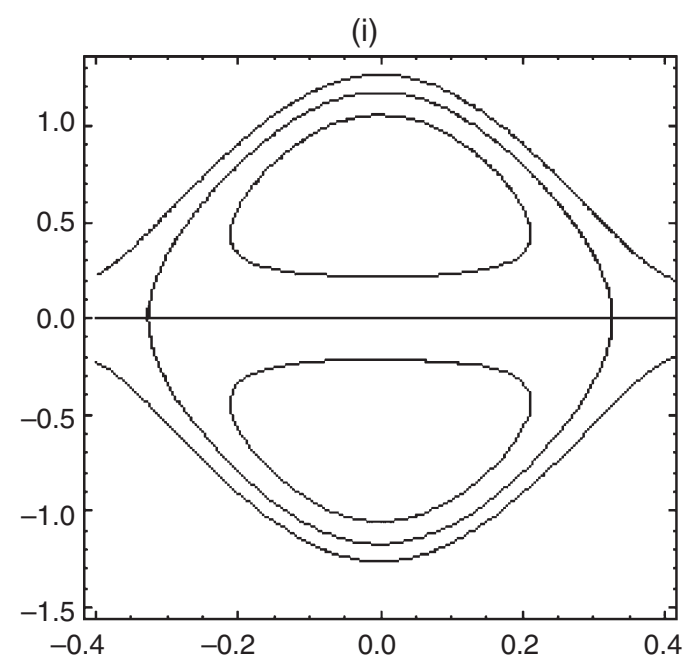

(ii)

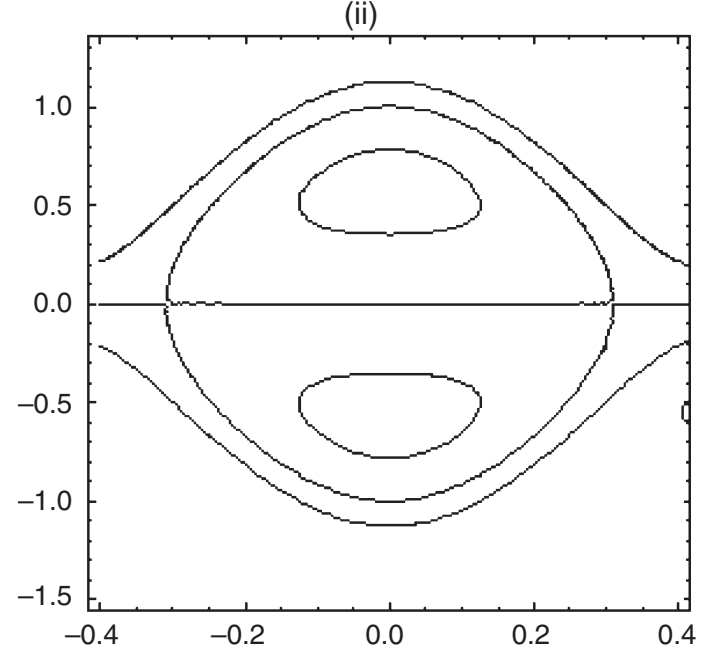

(iii)

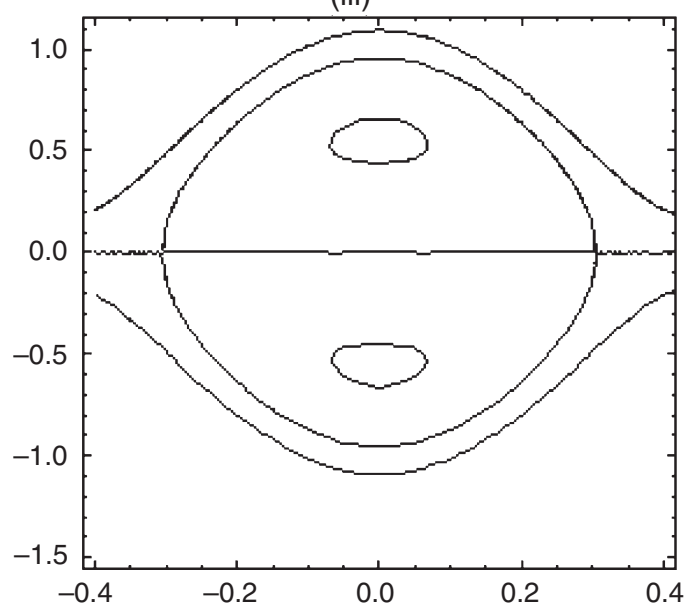

Fig. 9. Streamlines for $a=0.5, b=0.5, d=1 . \bar{Q}=1.4$ and $\beta=0.1$ for different $y$ (i) $y=0.3$, (ii) $y=0.5$, (iii) $y=0.7$.

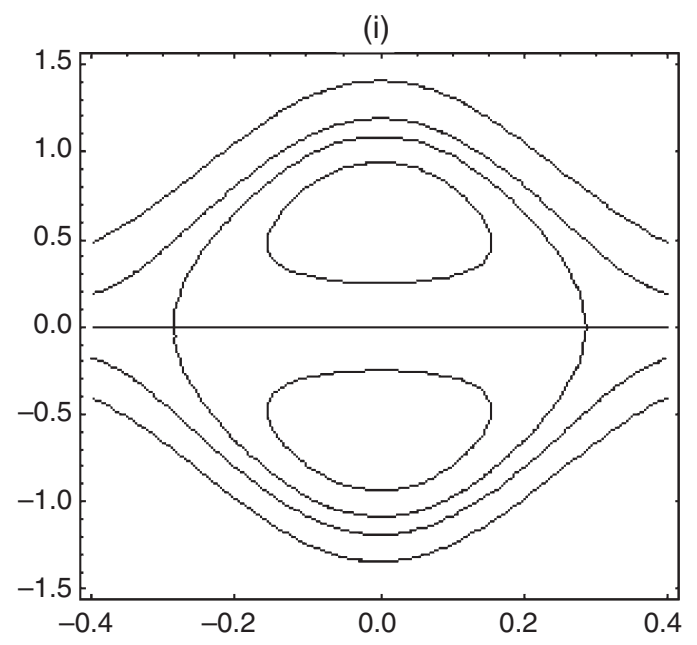

(ii)

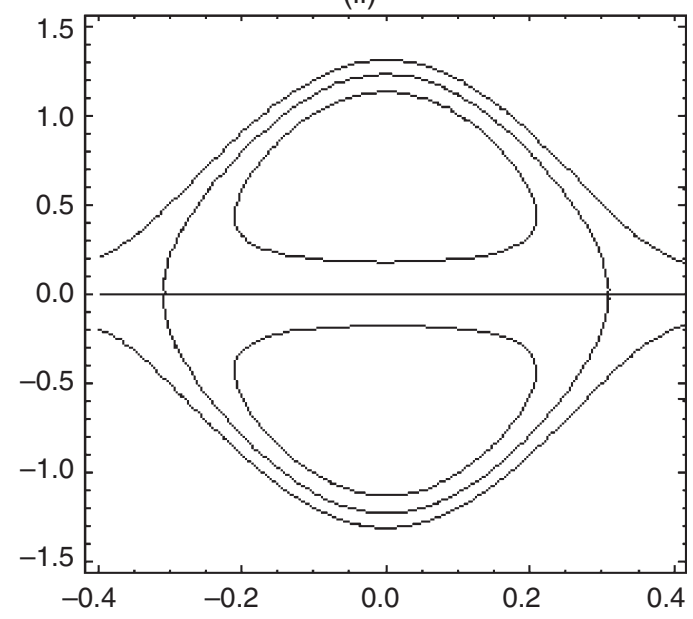

(iii)

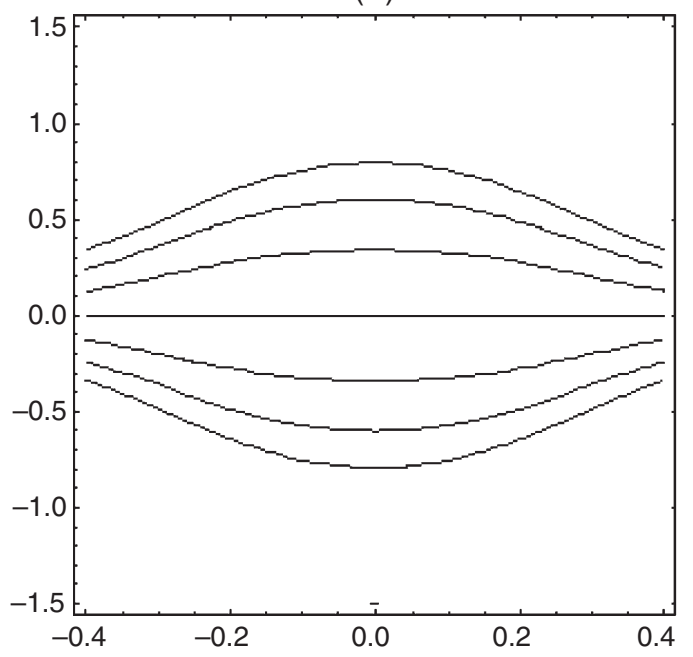

Fig. 10. Streamlines for $a=0.5, b=0.5, d=1 ; \bar{Q}=1.4$ and for different $\beta$ (i) $\beta=0.01$, (ii) $\beta=0.1$, (iii) $\beta=1$. 
(i)

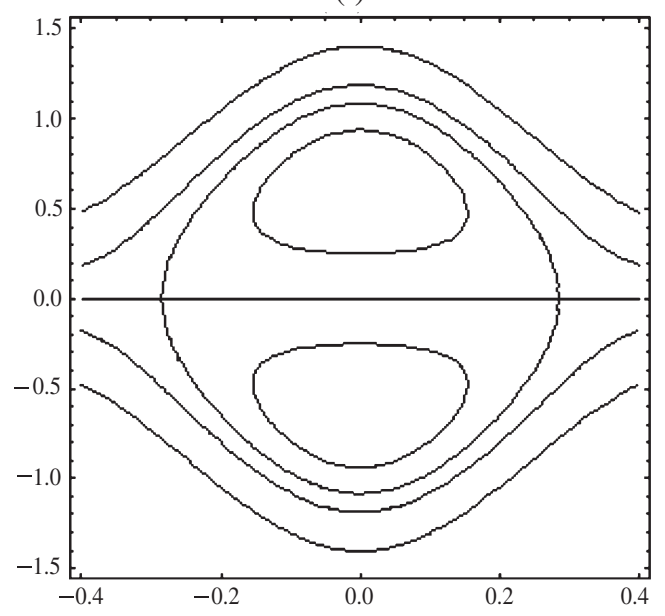

(ii)

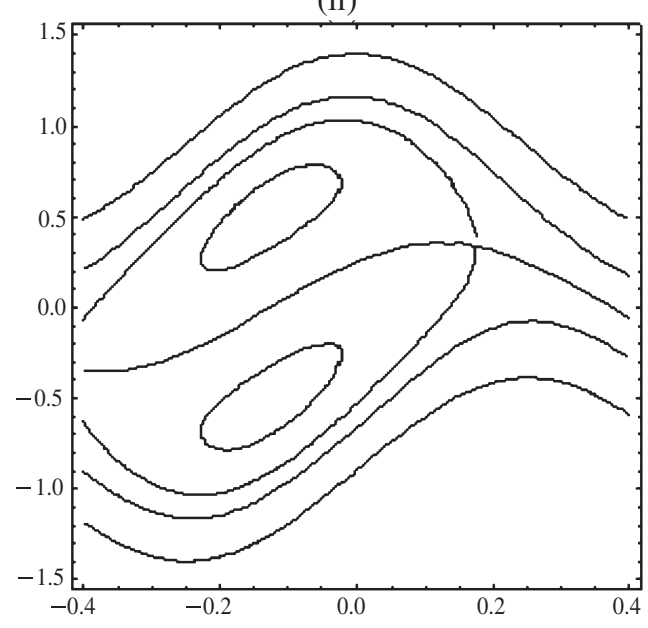

(iii)

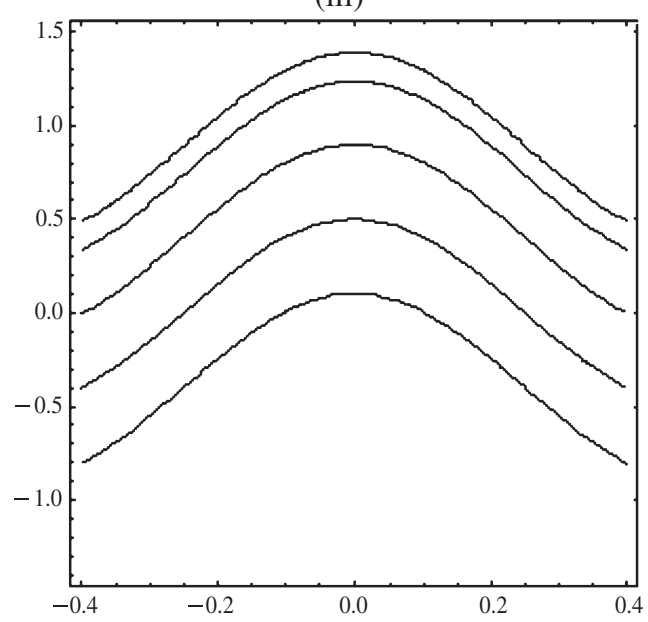

Fig. 11. Streamlines for $a=b=0.5 ; d=1 . \bar{Q}=1.4$ and $\beta=0.3$ for different $\gamma$ (i) $\gamma=0$, (ii) $\gamma=\pi / 2$, (iii) $\gamma=\pi$. sible only when $\Delta p<0$ corresponding to Poiseuille flow.

\section{Concluding remarks}

Peristaltic transport of an incompressible viscous fluid due to an asymmetric waves propagating on the horizontal sidewalls of a rectangular duct is studied under long-wavelength and low-Reynolds number approximations. The expressions of the axial velocity, axial pressure gradient and stream function are developed. Numerical integration is used to analyze the pumping characteristics. Stream lines are plotted to discuss the phenomena of trapping. Such a problem is important in understanding the motion of the intrauterine fluid in a sagittal cross section of the uterus. However, the sagittal cross section of the uterus may be better approximated by a tube of rectangular cross section than a two-dimensional channel. The present study discloses some important finding. These are

- The pressure gradient is higher for a square duct $(\beta=1)$ than that for a two dimensional channel.

- The pressure gradient (or the pressure rise) for a symmetric walls is higher than that for an asymmetric walls.

- The finite extent of the width of the duct alters the pumping characteristics.

- The pressure rise is higher for $\beta>1$ (a duct with a height large compared to the width) than that for $\beta=1$ (a square duct) than that for $\beta<1$ (rectangular duct) than that for $\beta=0$ (two-dimensional channel).

- The size of the trapped bolus increases with an increase in the aspect ratio $\beta$, while it decreases as the lateral station y increases.

- Symmetric channel gives bigger trapping zone.

- Future works can be done by using numerical methods to solve the governing equations of the problem without any approximation.

\section{References}

[1] A.H. Shapiro, M.Y. Jaffrin and S.L. Weinberg, Peristaltic pumping with long wavelength at low reynolds number, J Fluid Mech 37 (1969), 799.

[2] L.M. Srivastava and V.P. Srivastava, Peristaltic transport of blood: Casson model-II, J Biomech 17 (1984), 821.

[3] T. Hayat, Y. Wang, A.M. Siddiqui, K. Hutter and S. Asghar, Peristaltic transport of a third order fluid in a circular cylindrical tube, Math, Models Methods Appl Sci 12 (2002), 1691. 
[4] Kh.S. Mekheimer, Non-linear peristaltic transport of magneto-hydrodynamic flow in an inclined planar channel, Arab J Sci Eng 2A (2003), 183.

[5] Kh.S. Mekheimer and T.H. Al-Arabi, Nonlinear peristaltic transport of MHD flow through a porous medium, Int J Math and Math Sci 26 (2003), 1663.

[6] T. Hayat, Y. Wang, A.M. Siddiqui and K. Hutter, Peristaltic motion of Johnson-Segalman fluid in a planar channel, Math Probl Eng 1 (2003), 1.

[7] Kh.S. Mekheimer, Non-linear peristaltic transport through a porous medium in an inclined planar channel, J Porous Media 3 (2003), 189.

[8] Kh.S. Mekheimer, Peristaltic flow of blood under effect of a magnetic field in a non uniform channels, Appl Math Computation 153 (2004), 763.

[9] T. Hayat, Y. Wang, A.M. Siddiqui and K. Hutter, Peristaltic transport of an Oldroyd-B fluid in a planar channel, Math Problems Eng 4 (2004), 347.

[10] T. Hayat, F.M. Mahomed and S. Asghar, Peristaltic flow of magnetohydrodynamic Johnson-Segalman fluid, Nonlinear Dynamics 40 (2005), 375.

[11] M. Elshahed and M.H. Haroun, Peristaltic transport of JohnsonSegalman fluid under effect of a magnetic field, Math Probl Eng 6 (2005), 663.

[12] T. Hayat, M. Khan, S. Asghar and A.M. Siddiqui, A mathematical model of peristalsis in tubes through a porous medium, J Porous Media 9 (2006), 55

[13] T. Hayat and N. Ali, Peristaltically induced motion of a MHD third-grad fluid in a deformable tube, Physica A 370 (2006), 225.

[14] M.H. Haroun, Non-linear peristaltic flow of a fourth grade fluid in an inclined asymmetric channel, Comput Mater Sci 39 (2007), 324

[15] T. Hayat, M. Khan, A.M. Siddiqui and S. Asghar, Non-linear peristaltic flow of a non-Newtonian fluid under effect of a magnetic field in a planar channel, Commun in Nonlinear Sci and Numerical Simu 12 (2007), 910.

[16] M.H. Haroun, Effect of Deborah number and phase difference on peristaltic transport of a third-order fluid in an asymmetric channel, Nonlinear Sci Numer Simul 12 (2007), 1464.
[17] T. Hayat and N. Ali, Hall effects on peristaltic flow of a Maxwell fluid in a porous medium, Physics Letters A 363 (2007), 397.

[18] Kh.S. Mekheimer and Y.A. Abd el Mabod, The influence of heat transfer and magnetic field on peristaltic transport of a Newtonian fluid in a vertical annulus: Application of an endoscope, Physics letters A 372 (2008), 1657.

[19] N. Ali, Q. Hussain, T. Hayat and S. Asghar, Slip effects on the peristaltic transport of MHD fluid with variable viscosity, Physics Letters A 372 (2008), 1477.

[20] Kh.S. Mekheimer, Effect of the induced magnetic field on peristaltic flow of a couple stress fluid, Physics Letters A 372/23 (2008), 4271.

[21] K. De Vries, E.A. Lyons, J. Ballard, C.S. Levi and D.J. Lindsay, Contraction of the inner third myometrium, Am J Obstet Gynecol 162 (1990), 679.

[22] O. Eytan and D. Elad, Analysis of intra-uterine fluid motion induced by uterine contractions, Bull Math Biology 61 (1999), 221 .

[23] M. Mishra and A.R. Rao, Peristaltic transport of a Newtonian fluid in an asymmetric channel, $Z$ angew Math Phys $\mathbf{5 4}$ (2003), 532.

[24] M. Kothandapani and S. Srinivas, Non-linear peristaltic transport of a Newtonian fluid in an inclined asymmetric channel through a porous medium, Physics Letters A 372 (2008), 1265 .

[25] N. Ali and T. Hayat, Peristaltic flow of a micropolar fluid in an asymmetric channel, Computers and Mathematics with Applications $\mathbf{5 5}$ (2008), 589

[26] M.E. Erdogan, The effects of side walls on axial flow in rectangular ducts with suction and injection, Acta Mechanica 162 (2003), 157.

[27] S. Tsangaris, and N.W. Vlachakis, Exact solution of the navier-stokes equations for the fully developed, pulsating flow in a rectangular duct with constant cross-sectional velocity, ASME J Fluids Eng 125 (2003), 382.

[28] M.V.S. Reddy, M. Mishra, S. Sreenadh and A.R. Rao, Influence of lateral walls on peristaltic flow in a rectangular duct, Journal of Fluids Engineering 127 (2005), 824. 

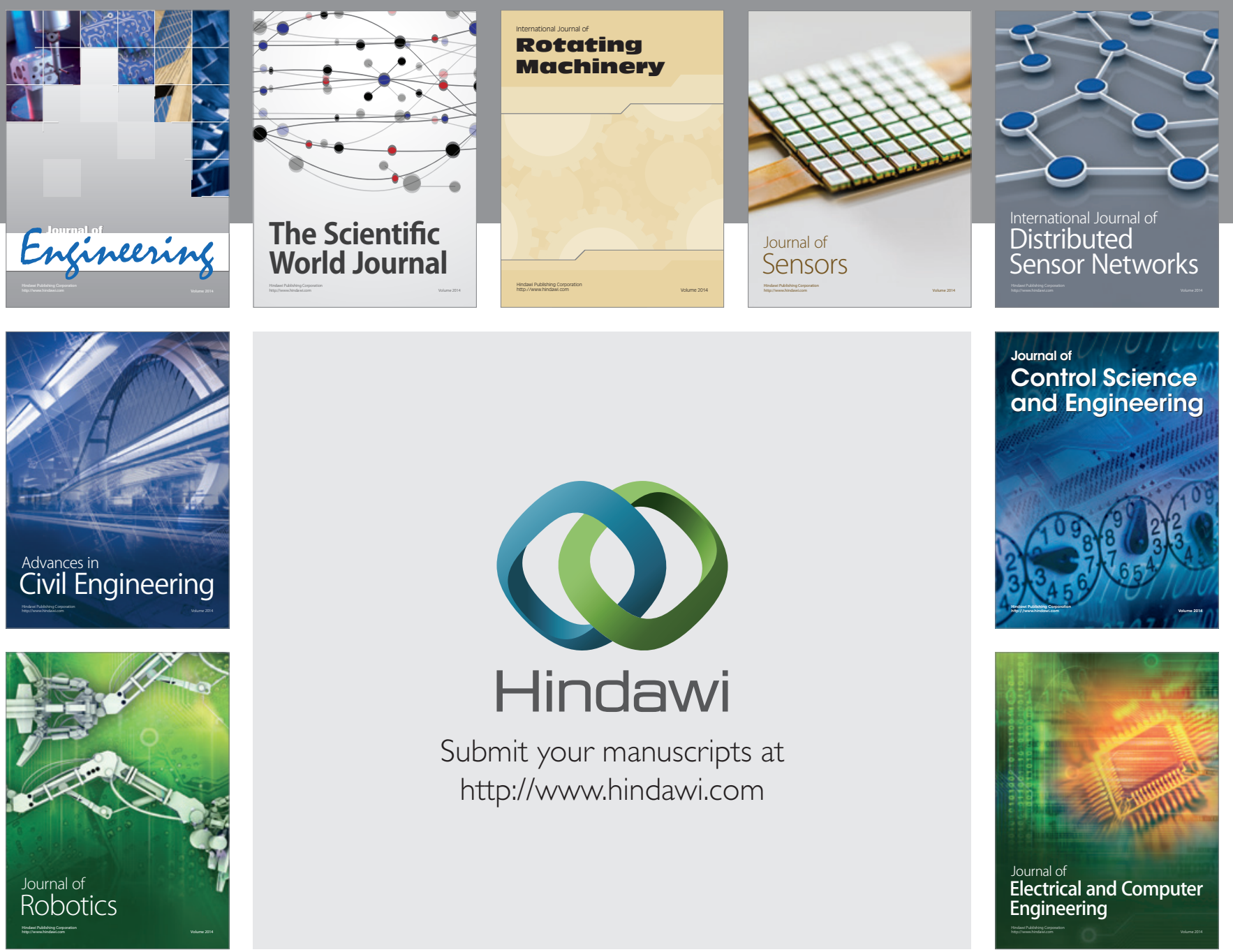

Submit your manuscripts at

http://www.hindawi.com
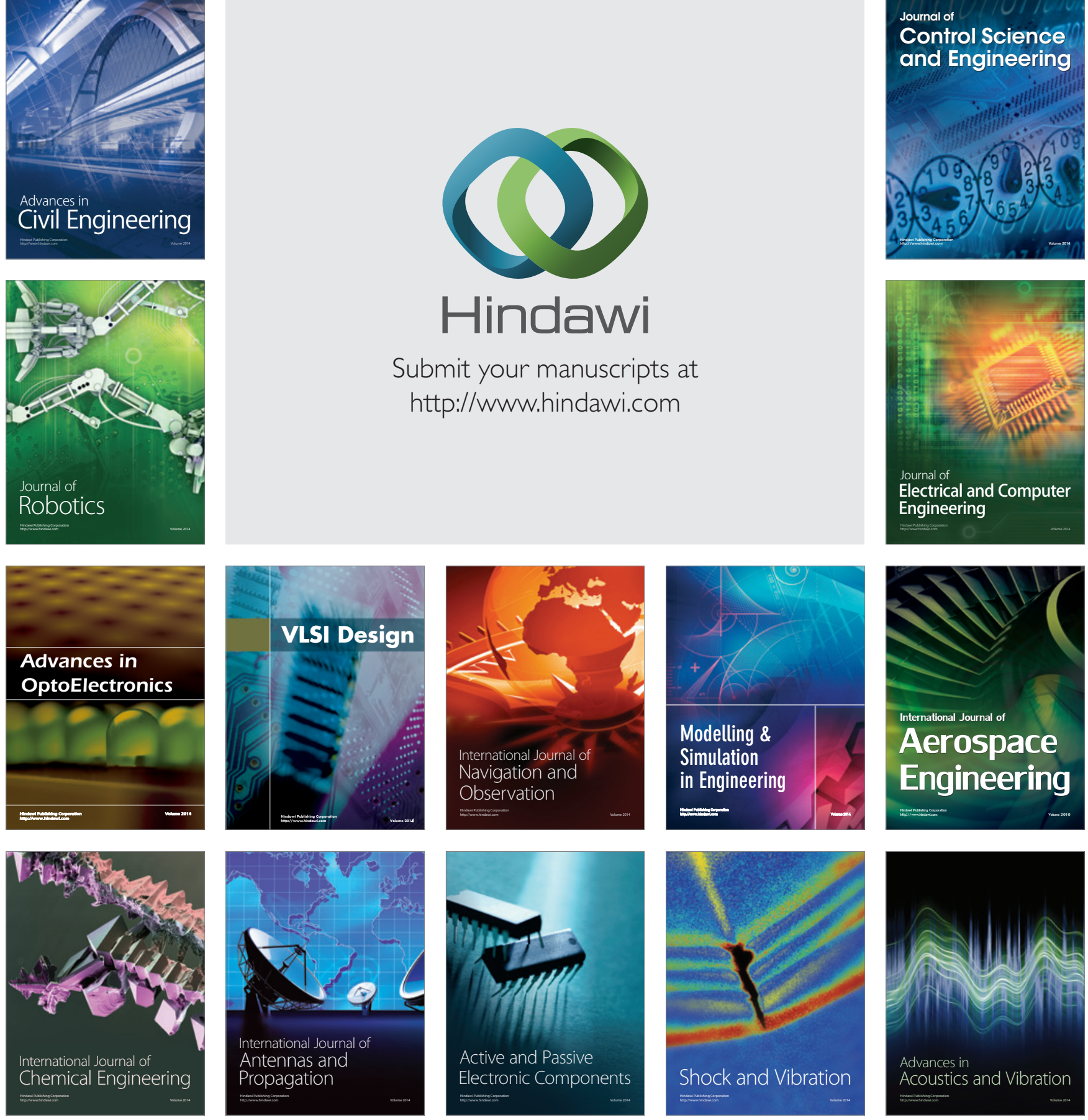